\title{
Empfehlungen aktueller Referenzwerte für die Spirometrie und Ganzkörperplethysmografie*
}

\author{
Recommendation of New Reference Values for Spirometry and \\ Body Plethysmography
}

Autor

Institut

\section{Baur}

Institut für Arbeitsmedizin (komm. Leiter: Prof. Dr. med. A. Fischer) Charité Universitätsmedizin Berlin eingereicht $\quad 8.5 .2013$

akzeptiert 31.5 .2013

Bibliografie

DOI http://dx.doi.org/

$10.1055 / \mathrm{s}-0033-1344331$

Pneumologie 2013; 67: 401-405

(c) Georg Thieme Verlag KG

Stuttgart · New York

ISSN 0934-8387

Korrespondenzadresse

Prof. Dr. med. Xaver Baur Institut für Arbeitsmedizin Charité Universitätsmedizin Berlin

Thielallee 69

14195 Berlin

Xaver.baur@charite.de

\section{Zusammenfassung \\ $\nabla$}

Hintergrund und Methode: Ende 2012 veröffentlichte eine Task Force der ERS nach fünfjähriger Arbeit auf Basis einer integrativen Auswertung der zur Verfügung stehenden, nach anerkannten Qualitätskriterien durchgeführten Studien neue Referenzwert-Empfehlungen für die Spirometrie (jeweils Mittel- und Grenzwert).

Ergebnisse und Schlussfolgerungen: Es handelt sich um eine der bisher umfangreichsten derartigen Auswertungen in der Pneumologie (über 74.000 gesunde Probanden vom 3. bis 95.Lebensjahr). Im Vergleich zu den bisher vorherrschend verwendeten EGKS-Referenzwerten ergeben sich insbesondere im höheren Lebensalter Abweichungen um ca. 10\% nach oben. Es wird empfohlen, diese Referenzwerte mit neueren Referenzwerten der Ganzkörperplethysmografie zu verbinden und anstelle der nicht unserer heutigen Population entsprechenden EGKS-Empfehlungen 1983/1993 zu verwenden.

\section{Einleitung}

$\nabla$

Die bisher in Europa überwiegend verwendeten Lungenfunktionsreferenzwerte [1,2], 1993 als unveränderte Wiederauflage von 1983 publiziert, gehen auf Reihenuntersuchungen in den 1960erund 1970er-Jahren zurück, die nicht heutigen epidemiologischen und biostatistischen Anforderungen entsprechen. Beispielsweise wurden die Referenzwerte der Frauen von Messwerten der Männer abgeleitet; auch sind die Randbereiche (des Alters, der Körpergröße) nur spärlich besetzt. Im Folgenden sollen neue Referenzwert-Studien, die diese Limitierungen nicht oder nicht in diesem Maße haben, vorgestellt und abschließend

* basierend auf einem Vortrag auf der DGP-Jahrestagung Hannover 2013.

\section{Abstract \\ $\nabla$}

Background and methods: At the end of 2012, after 5 years of work, a task force of the ERS published new reference values for spirometry (mean values and Lower Limits of Normal, LLN) based on available studies, performed according to generally accepted quality criteria.

Results and conclusions: So far this is one of the most comprehensive work on reference values in pneumology (more than 74,000 healthy subjects, age range 3 to 95 years). These reference values are approximately $10 \%$ higher (especially in the elderly population) than the still preferentially used ECCS reference values. It is recommended to combine and use them in combination with new reference values for body plethysmography by replacing the ECCS recommendations of 1983/1993 which show discrepancies to lung function data of our today population.

Empfehlungen für deren Verwendung ausgesprochen werden.

\section{Ergebnisse \\ $\nabla$}

Referenzwerte der Global Lung Initiave (GLI)

Basierend auf Messungen von über 74.000 gesunden Probanden erarbeitete die ERS-Task Force Global Lung Initiative (Chairs: J. Stocks, X. Baur, G. Hall, B. Culver) in den letzten fünf Jahren neue spirometrische Referenzwerte, die kürzlich unter Federführung von Quanjer veröffentlicht wurden [3]. Dabei wurden die Daten von 72 Studien, die nach Angaben der Untersucher entsprechend international anerkannten Qualitätskriterien durchgeführt wurden, berücksichtigt [1]. 


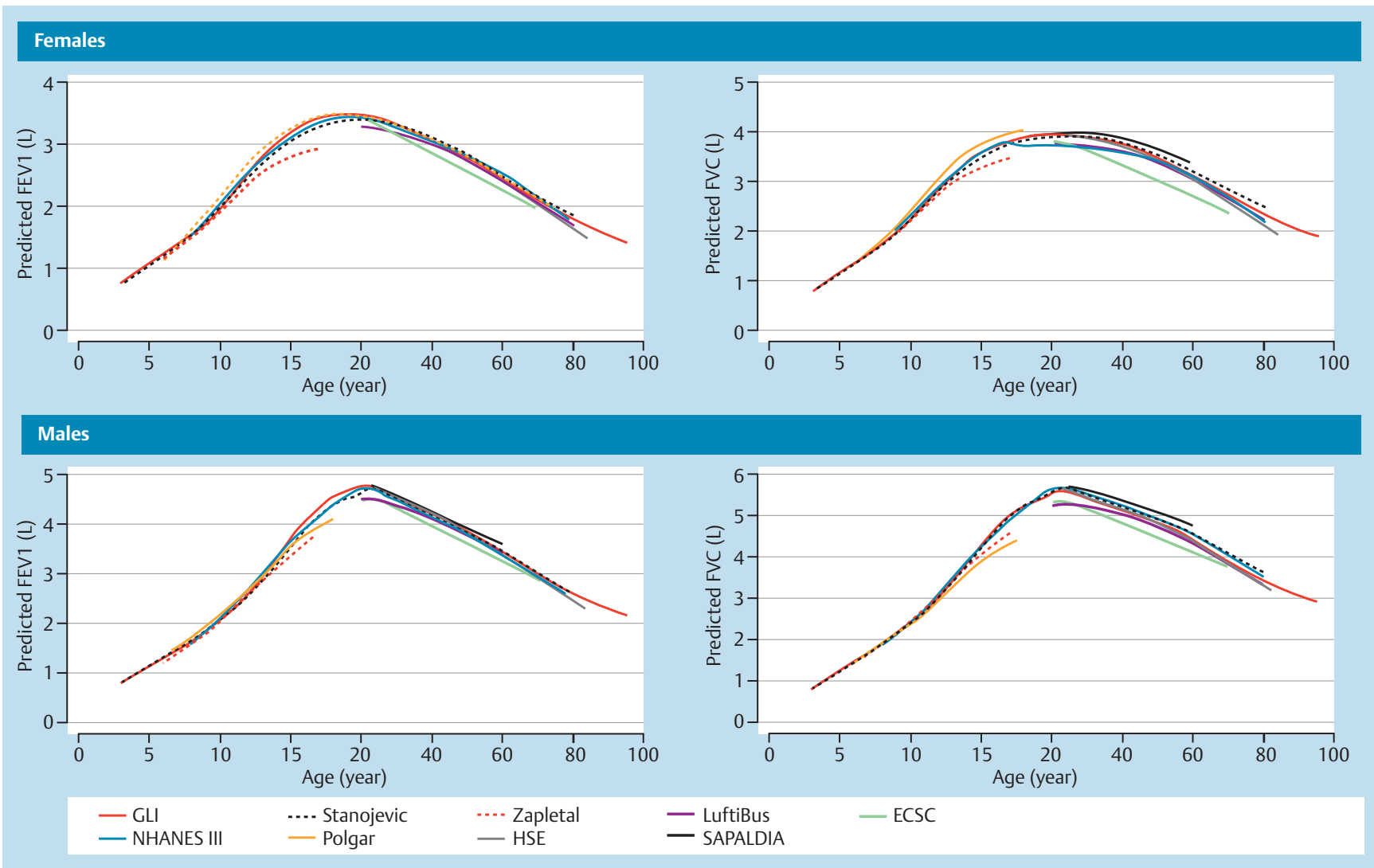

Abb. 1 Vergleich der FEV 1 - und der FVG-Sollmittelwerte für Frauen (oben) und Männer (unten) in verschiedenen Studien. GLI-Vorläufer =Global Lung Initiative [4], NHANES III (Hankinson, Odencrantz et al. 1999 [5]), ECSC [6] (European Community for Steel and Coal ) [1] , HSE (Health Survey for England 1995 - 1996) [6], LuftiBus [7], SAPALDIA [8], Polgar und Promadhat [9], Zapletal et al. [10].

Die integrativ daraus abgeleiteten aktuellen Referenzwerte bilden nahezu die gesamte Lebensspanne (von 3-95 Jahren) kontinuierlich ab. Auch für Frauen liegt eine ausreichende Zahl von Messwerten zugrunde. Es handelt sich um die ersten multiethnischen spirometrischen Lungenfunktionsreferenzwerte. Sie umfassen Kaukasier (einschließlich Europäer, Israelis, Australier, US-Amerikaner mexikanischer Herkunft und Einwohner der südamerikanischen Staaten Brasilien, Chile, Mexiko, Uruguay, Venezuela, Algeriens und Tunesiens), US-Amerikaner afrikanischer Abstammung, die Bevölkerung Südost-Asiens (einschließlich des südlichen Chinas, Taiwans und Thailands) sowie Nordost-Asiens (einschließlich Koreas und des nördlichen Chinas).

Für folgende Parameter wurden neue Referenzgleichungen erstellt: $\mathrm{FVC}, \mathrm{FEV}_{1}, \mathrm{FEV}_{1} / \mathrm{FVC} \mathrm{FEF}_{25-75}, \mathrm{FEF}_{75}$ und zusätzlich für den Altersbereich unter 10 Jahre: $\mathrm{FEV}_{0.75}, \mathrm{FEV}_{0.75} / \mathrm{FVC}$. Dabei wurde ein LMS-Algorithmus angewendet, wobei „L“ für die Krümmung des Sollwertverlaufs, „M“ für den Mittelwert und „S“ für den Variationskoeffizienten steht. Alle Parameter sind Funktionen von Geschlecht, Alter und Körpergröße sowie ethnischer Zugehörigkeit. Einzelheiten s. Anhang.

FVC und FEV 1 der GLI 2012 liegen im mittleren und höheren Lebensabschnitt ca. $10 \%$ höher [2] als nach den bisher favorisierten Referenzwertempfehlungen [1] ( $\bullet$ Abb.1).

- Abb.2 gibt die Unterschiede zwischen verschiedenen ethnischen Gruppen wieder. Es besteht die Möglichkeit, die vorgenannten Referenzwerte als Basis für die Ermittlung neuer Referenzwerte zusätzlicher Gruppen zu verwenden; Einzelheiten hierzu finden sich unter www.lungfunction.org

\section{Unterer Grenzwert (LLN) und der Z-Score}

Nach EGKS 1983/1993 [1] wird über den gesamten Altersbereich vom Referenz-Mittelwert einheitlich das 1,64-fache der im Gesamtkollektiv ermittelten Standardabweichung abgezogen (95\%Vertrauensbereich, LLN), um den Grenzwert zu erreichen. Vielfach wird noch gröber vorgegangen und $80 \%$ bzw. $70 \%$ des Referenzmittelwertes der VC und der FEV 1 als Grenzwert definiert.

Die Auswertungen der GLI 2012 zeigen aber, dass die Streuung der Messwerte weder einem fixen Wert noch einer einheitlichen relativen Abweichung vom Referenz-Mittelwert entspricht, sondern in den Randbereichen größer als im mittleren Altersabschnitt ist ( $\boldsymbol{A}$ Abb.3). Nach GLI 2012 werden daher der untere Grenzwert (LLN) und der Z-Score für jeden Altersabschnitt ebenfalls per L, $M$ und $S$ ermittelt. Der Z-Score für den einzelnen Messwert wird erhalten durch Division der Differenz zwischen Messwert und Referenz-Mittelwert durch die Standardabweichung des Referenzkollektives (Messwert-Referenz-Mittelwert/SD).

\section{Weitere neuere Lungenfunktionsreferenzwerte}

Die Messdaten der 1943 gesunden Kinder (Alter von 4-18 Jahre) der LUNOKID-Studie [11] flossen in die GLI-Auswertung ein; es gibt bis auf eine geringere Variabilität und diskret höhere Werte der 4-8-Jährigen keine wesentlichen Abweichungen.

Auch die Untersuchung von 686 bzw. 1302 gesunden erwachsenen Nichtrauchern zeigt hiervon keine größeren Abweichungen $[12,13]$. Die zusätzlichen ganzkörperplethysmografischen Parameter TLC, RV, $\mathrm{FRC}_{\text {pleth }}$ und RV/TLC der letzteren ReferenzwertErhebungen weichen etwa um denselben Anteil von den EGKSReferenzwerten nach oben ab wie die spirometrischen GLI- 

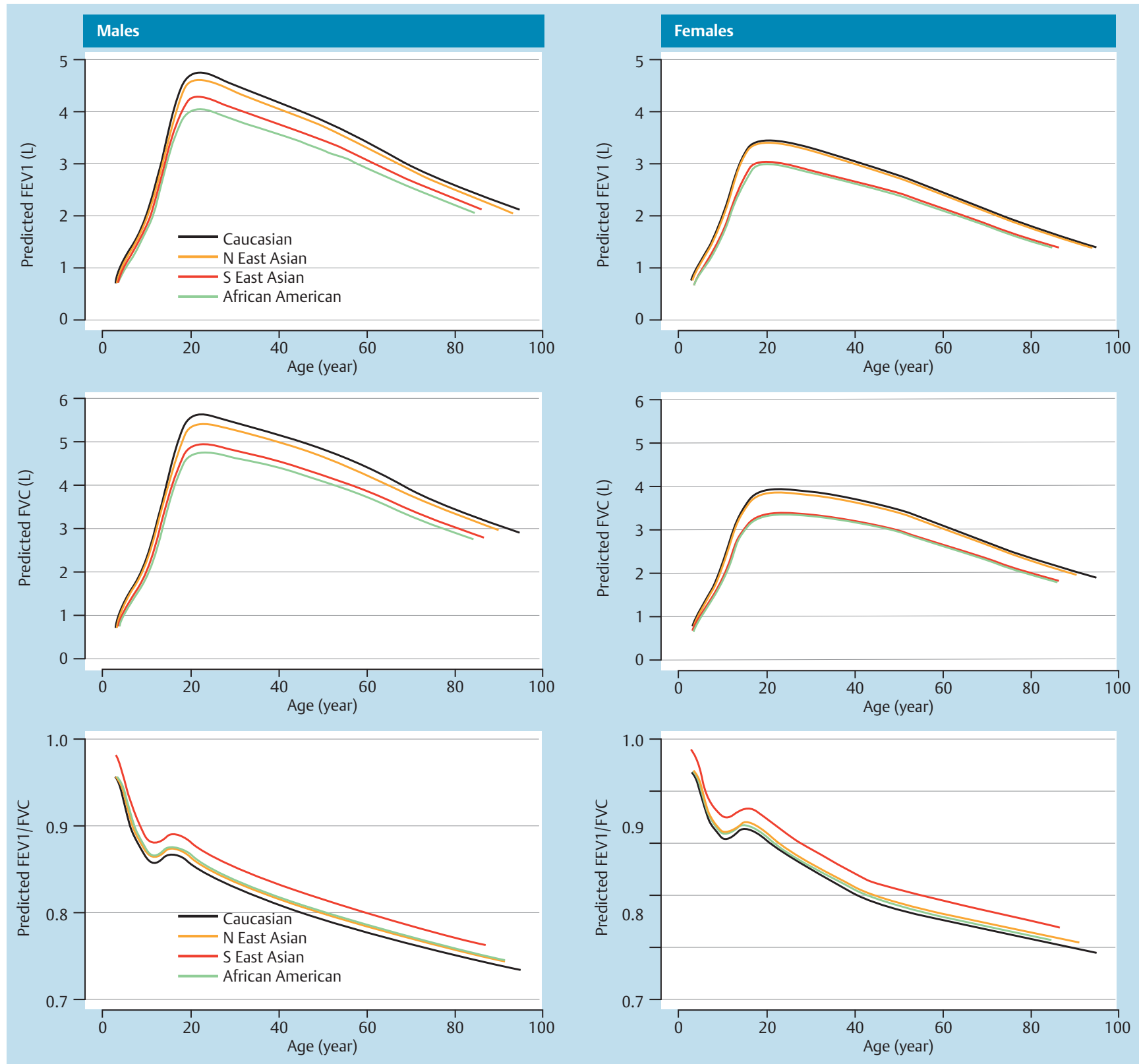

Abb.2 Neue Referenzwerte für FVC, FEV 1 , FEV $/$ /FVC verschiedener ethnischer Gruppen. Nachdruck mit freundlicher Genehmigung der European Respiratory Society [3].

Daten. Für die Resistance ergibt sich ein oberer Grenzwert des erwachsenen Mannes von etwa $0,3 \mathrm{kPa} \cdot \mathrm{L}^{-1}$, der Frau von $\left.0,35 \mathrm{kPa} \cdot \mathrm{s} \cdot \mathrm{L}^{-1}\right)$.

\section{Schlussfolgerung und Empfehlungen}

Die kürzlich publizierten, bisher umfangreichsten Lungenfunktion-Referenzwerte der GLI 2012 basieren auf den Messdaten neuerer, nach anerkannten Qualitätskriterien durchgeführten Studien an gesunden Nichtrauchern. Sie weisen im Vergleich zu den bisher überwiegend verwendeten EGKS-Referenzwerten [1, 14,15] für die Vitalkapazität und $\mathrm{FEV}_{1}$ im Mittel knapp $10 \%$ höhere Werte aus, d.h., sie sind sensitiver [2]. Sie entsprechen am ehesten der heutigen Bevölkerung und decken nahezu den gesamten Lebensbereich kontinuierlich ab. Außerdem umfassen sie verschiedene ethnische Gruppen. Für die Erstuntersuchung

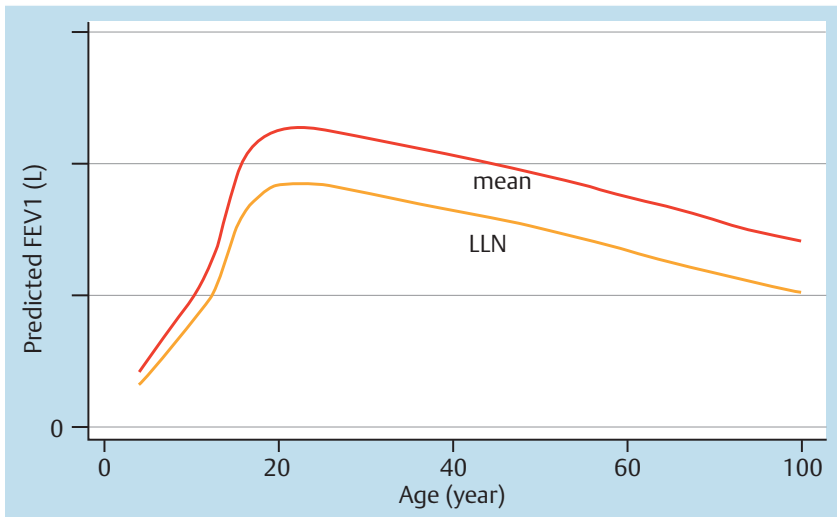

Abb. 3 Altersabhängigkeit der Referenzmittelwerte (mean) und -grenzwerte (LLN) der FEV 1 (männliches Geschlecht) [3]. Beachte, dass die Differenz der beiden kein fixer Anteil ist. 


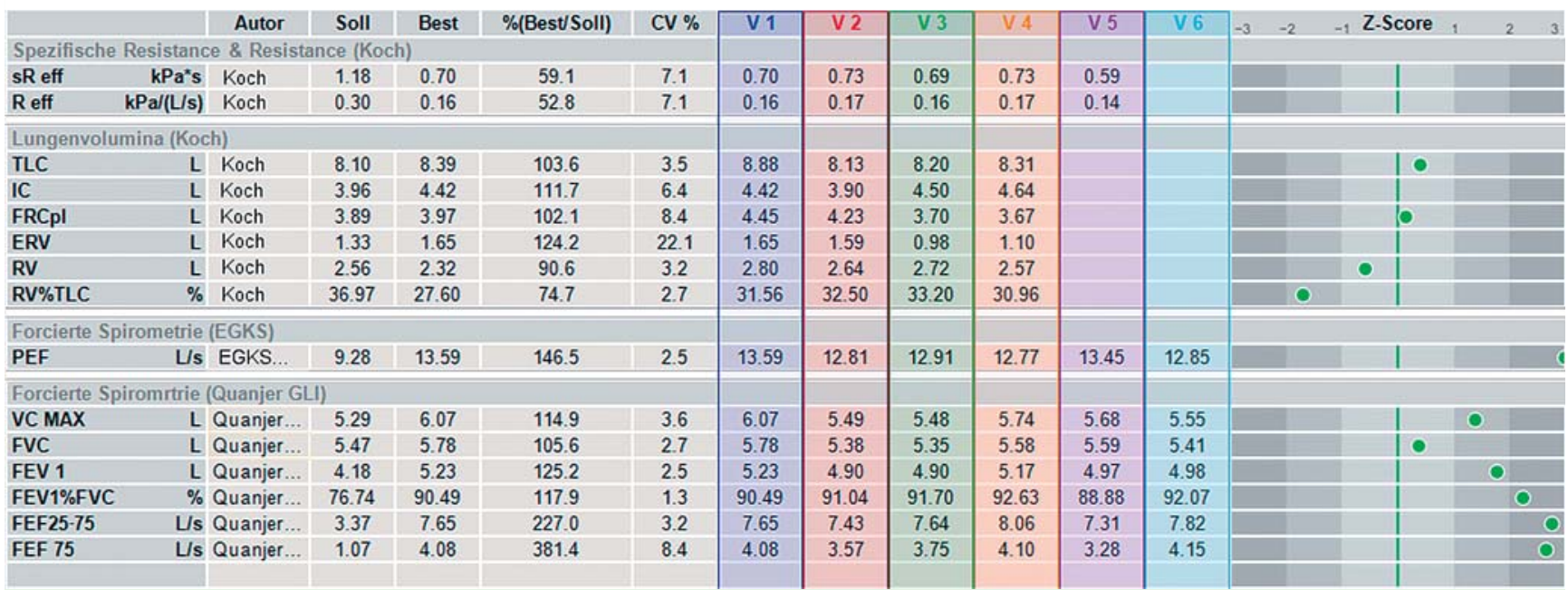

Abb.4 Befundausdruck (nummerisch und mittels Z-Score) der Lungenfunktionsdaten eines Probanden anhand der empfohlenen Referenzwerte; Einzelheiten siehe Text.

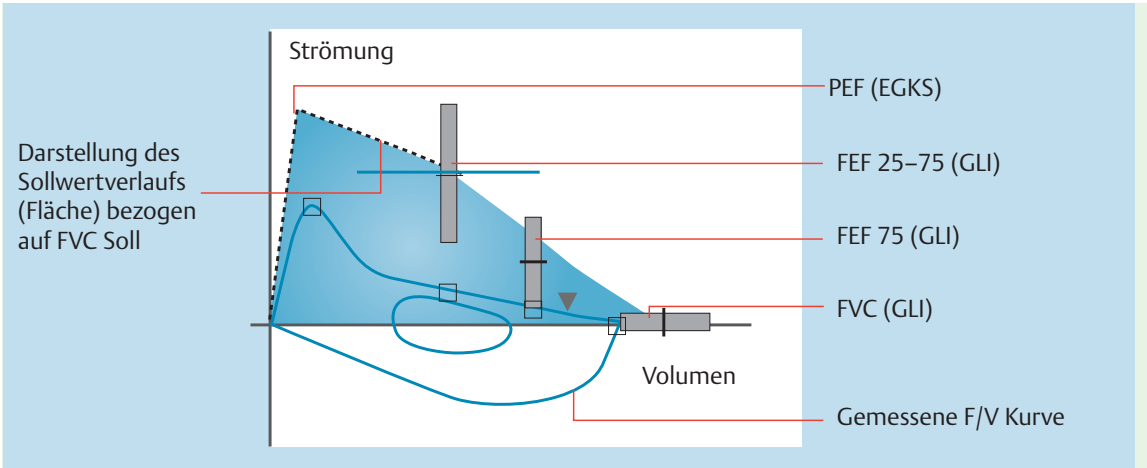

Abb.5 Empfohlene Darstellung der Fluss-Volumen-Kurse. Einzelheiten siehe Text. (H.-J. Smith) EGKS [1], GLI [3].

wird eine Orientierung am individuellen Grenzwert, dem ZScore von -1,64 (Mittelwert-1,64×SD) empfohlen, womit eine korrekte Interpretation ermöglicht wird. Zur Graduierung der Abweichungen der spirometrischen Parameter und Lungenvolumina (bei letzteren ist im Gegensatz zu ersteren eine beidseitige Betrachtung erforderlich) wird vorgeschlagen ( $\bullet$ Tab.1):

Tab. 1 Empfohlene Graduierung der Lungenfunktions-Messwertabweichungen vom jeweiligen Referenzwert unter Berücksichtigung des Z-Scores.

\begin{tabular}{ll}
\hline Normalbereich & \multicolumn{1}{c}{ Messwert $\geq-1,645$ Z-Score (LLN) } \\
\hline leichte Einschränkung & $-1,645>$ Messwert $\geq-1,960$ Z-Score \\
\hline mittelgradige/schwere & $-1,960>$ Messwert \\
Einschränkung &
\end{tabular}

- 1,645 Z-Score=5. Perzentil; - 1,960 Z-Score=2,5. Perzentil

Es ist davon auszugehen, dass sich diese neuen Referenzwerte in nächster Zeit weltweit durchsetzen werden. Aktuell implementiert sie bereits ein Großteil der Gerätehersteller, wobei sich noch kein einheitliches Vorgehen abzeichnet.

Die bisher verwendeten Referenzwerte für TLC, RV, RV/TLC, FRC, PEF, $\mathrm{FEF}_{50}$ und $\mathrm{FEF}_{25}$ von Quanjer et al. 1993 [1] sind mit den neuen GLI-Referenzwerten nicht kompatibel. Plausibel und kompatibel sind dagegen für erwachsene Kaukasier die Lungenvolumina und ganzkörperplethysmografischen Resistance-Werte von Koch et al. 2013 [12].

- Abb. 4 fasst im Sinne einer sensitiven und bestmöglichen Interpretation von Lungenfunktionsdaten eine sinnvolle Kombination aktueller Lungenfunktionsreferenzwerte zusammen (Einschrän- kungen liegen im Vergleich zu den bisherigen EGKS-Empfehlungen dabei früher bzw. tiefer im pathologischen Bereich). Da GLI 2012 bisher keine Referenzwerte für PEF, $\mathrm{FEF}_{25}$, und $\mathrm{FEF}_{50}$ liefert, bietet es sich an, Letztere bei Bedarf aus Quanjer et al. (1993) [1] zu übernehmen.

Vorschlag für eine einheitliche und optimierte grafische Darstellung der Spirometrie-Befunde

Aktuell werden von den Herstellern der Lungenfunktionsgeräte unterschiedliche, einen Vergleich und oft auch eine Blickdiagnose erschwerende grafische Wiedergaben der Spirometrie editiert. Auch ist es bisher überwiegend üblich, hierbei in der FlussVolumen-Kurve die Referenz-Mittelwerte und -Streubereiche der exspiratorischen Flüsse auf die Referenz-Vitalkapazität und nicht auf die sinnvollerweise zu verwendende Ist-Vitalkapazität zu beziehen. Es wird die in Abb. 5 dargestellte grafische Befundwiedergabe empfohlen. Dabei ist zu beachten, dass GLI 2012 [3] keine Empfehlungen für $\mathrm{PEF}, \mathrm{FEF}_{50}$ und $\mathrm{FEF}_{25}$ ausspricht, sodass hierfür bei Bedarf weiterhin die EGKS-Referenzwerte von 1983/1993 [1] empfohlen werden.

\section{Procedere}

Es wird ein innerhalb der ERS abgestimmtes Vorgehen empfohlen, sodass europaweit ein einheitliches Beurteilungssystem Anwendung findet, wodurch Abweichungen in verschiedenen europäischen Zentren vermieden und auch wissenschaftliche Kooperationen erleichtert würden. 


\section{Anhang}

LLN $($ 5er Perzentil $)=\exp (\ln (\mathrm{M})+\ln (1-1.645 \cdot \mathrm{L} \cdot \mathrm{S}) / \mathrm{L})$

Z-Score $=\left((\text { Messwert } / \mathrm{M})^{\mathrm{L}}-1\right) /(\mathrm{L} \cdot \mathrm{S})$

Die Volumina werden in Liter, das Alter (Age) in Jahren und die Körpergröße (Height) in $\mathrm{cm}$ angegeben. Körpergröße und Alter sollten mit mindestens einer Dezimalstelle in diese Gleichung eingegeben werden.

Bezüglich der altersabhängigen linearen Interpolation von L-Spline, M-Spline, S-Spline s. "lookup tables" www.lungfunction.org/ For IT Engineers and Manufacturers. Hier finden sich Koeffizienten und anschauliche Sollwerttabellen für die Ermittlung der Referenzwerte einschließlich ihrer unteren 95\%-Vertrauensbereiche und der Z-Zonen. Instruktionen zur Implementierung der Gleichungen in die Software der Lungenfunktionsgeräte. Beispiele erhält man unter www.lungfunction.org/ Access Equations and Tools. Eine kostenlose Software zur routinemäßigen klinischen Anwendung und für Forschungszwecke, die auch Interpretationen von Lungenfunktionsmessdaten in Bezug auf die neuen Referenzwerte, der 95\%-Vertrauensbereiche und der Z-Zonen erlaubt, ist ebenfalls zugänglich, s. www.spirxpert.com/download/ - > InstallGLI2012DataConversion.exe

\section{Interessenkonflikt}

Der Autor gibt an, dass kein Interessenkonflikt besteht.

\section{Literatur}

1 Quanjer PH, Tammeling GJ, Cotes JE et al. Lung volumes and forced ventilatory flows. Report Working Party Standardization of Lung Function Tests, European Community for Steel and Coal. Official Statement of the European Respiratory Society. Eur Respir J Suppl 1993; 16: 5-40

2 Baur X. Crashkurs Lungenfunktion. München-Deisenhofen: DustriVerlag; 2013
3 Quanjer PH, Stanojevic S, Cole TJ et al. Multi-ethnic reference values for spirometry for the 3-95-yr age range: the global lung function 2012 equations. Eur Respir J 2012; 40: 1324-1343

4 Stanojevic S, Wade A, Cole TJ. Spirometry centile charts for young Caucasian children: the Asthma UK Collaborative Initiative. Am J Respir Crit Care Med 2009; 180: 547-552

5 Hankinson JL, Odencrantz JR, Fedan KB. Spirometric reference values from a sample of the general U.S. population. Am J Respir Crit Care Med 1999; 159: 179-187

6 Falaschetti E, Laiho J, Primatesta $P$ et al. Prediction equations for normal and low lung function from the Health Survey for England. Eur Respir J 2004; 23: 456- 463

7 Kuster SP, Kuster D, Schindler C et al. Reference equations for lung function screening of healthy never-smoking adults aged $18-80$ years. Eur Respir J 2008; 31: 860 - 868

8 Brandli $O$, Schindler $C$, Kunzli $N$ et al. Lung function in healthy never smoking adults: reference values and lower limits of normal of a Swiss population. Thorax 1996; 51: $277-283$

9 Polgar G, Promadhat V. Pulmonary function testing in children: Techniques and standards. Philadelphia: Saunders; 1971

10 Zapletal A, Paul T, Samanek N. Die Bedeutung heutiger Methoden der Lungenfunktionsdiagnostik zur Feststellung einer Obstruktion der Atemwege bei Kindern und Jugendlichen. Z Erkrank Atm-Org 1977; 149: $343-371$

11 Huls A, Kramer U, Gappa $M$ et al. [New spirometric reference values for children and adolescents in Germany considering height and non-linear age effects: the LUNOKID-study]. Pneumologie 2013; 67: 141 - 149

12 Koch B, Friedrich $N$, Volzke $H$ et al. Static lung volumes and airway resistance reference values in healthy adults. Respirology 2013; 18:170-178

13 Koch B, Schaper C, Ittermann $T$ et al. Reference values for respiratory pressures in a general adult population-results of the Study of Health in Pomerania (SHIP). Clin Physiol Funct Imaging 2010; 30: 460 - 465

14 Boesch $D$, Criee $C P$. Lungenfunktionsprüfung. Heidelberg: Springer-Verlag; 1999

15 Criée CP, Berdel D, Heise D et al. Lungenfunktion: Spirometrie, Atemmuskelfunktion. München: Dustri-Verlag Dr. Karl Feistle; 2006 